\title{
EFFICIENT DATA COMPRESSION OF ECG SIGNAL USING DISCRETE WAVELET TRANSFORM
}

\author{
Karishma Qureshi ${ }^{1}$, V. P. Patel ${ }^{2}$ \\ ${ }^{1}$ PG Student, ${ }^{2}$ Head of the Department, Applied Instrumentation, L. D. College of Engineering, Gujarat, India \\ qureshikarishma@gmail.com,vinodpatel_74@rediffmail.com
}

\begin{abstract}
Data compression reduces the number of bits of information required to store or transmit the biomedical signals. Compression algorithm of bio-medical signals is implemented using Discrete Wavelet Transform. High threshold value ( $\lambda$ ) gives high data reduction and poor signal fidelity and low threshold value $(\lambda)$ gives low data reduction and high signal fidelity. Threshold value selection should be such that the quality of the ECG signal is not distorted on reconstruction and a good amount of data reduction is also achieved. The database has been collected from MIT-BIH arrhythmias database of the lead II (ML II) signal. The ECG signal to be compressed is decomposed to the Level 5 using the biorthogonal 4.4 wavelet family. In this paper different issues for compression are demonstrated and the results are shown using MATLAB
\end{abstract}

Index Terms: Electrocardiogram (ECG), Discrete Wavelet Transform (DWT), Compression Ratio (CR), Percentage Root Mean Deviation (PRD), etc.

\section{INTRODUCTION}

There is an exponential increase in digital data, obtained from various signals specially the biomedical signals such as electrocardiogram (ECG), electroencephalogram (EEG), electromyogram (EMG) etc. How to transmit or store these signals efficiently becomes the most important issue. [1] Transmission techniques of these biomedical signals through communication channels is an important issue as it allows experts to make a remote assessment of the information carried by the signals, in a very cost-effective way. Storage of these bio-medical signals leads to a large volume of information. Thus the necessity of efficient data compression methods for biomedical signals is currently widely recognized. [3]

The compression of Electrocardiogram signals is done using Discrete Wavelet Transform (DWT). Thus, a digital compression technique is used to solve this problem. In this paper, the compression of ECG signal has been done with biorthogonal wavelet function. The parameters such as compression ratio and percentage root mean deviation are calculated for different samples of ECG signal. 1024, 2048, 4096, 8192, 16384 samples are taken for record 100 from MIT-BIH arrhythmias database and compression is done.

\section{ECG SIGNAL COMPRESSSION}

ECG compression techniques can be categorized into: direct time-domain techniques, transformed frequency domain techniques and parameters optimization techniques.[3]

\subsection{Direct Signal Compression Techniques}

A direct method performs the compression directly on the ECG signal. These are also known as time domain techniques. To get a high performance time domain compression algorithm, intelligent sample selection criteria should be used. The original signal is reconstructed by an inverse process, often by drawing straight lines between the extracted samples. The key to a successful algorithm is the development of a good rule for determining the most significant samples. [3]

\subsection{Transformed ECG Compression Methods}

Transform method, converts the time domain signal to the frequency or other domains and analyzes the energy distribution. These methods mainly utilize the spectral and energy distributions of the signal by means of some transform, and properly encoding the transformed output.

Signal reconstruction is achieved by an inverse transformation process. This category includes different transform techniques such as the Karhunen-Loève transform, Fourier transform, Cosine transform, sub band-techniques, vector quantization (VQ), and more recently the wavelet transform. Wavelet technique is the obvious choice for ECG signal compression because of its localized and non-stationary property and the well-proven ability of wavelets to see through signals at different resolutions. The main task in wavelet analysis (decomposition and reconstruction) is to find a good analyzing function (mother wavelet) to perform an optimal decomposition. [3] 


\subsection{Optimization Methods for ECG Compression}

Optimization method minimizes the reconstruction error given a bound on the number of samples to be extracted or the quality of the reconstructed signal to be achieved. The ECG signal is compressed by extracting the signal samples that, after interpolation, will best represent the original signal given an upper bound on their number. The aim is to present ECG compression technique that achieves maximum data volume reduction while preserving the significant signal morphology features upon reconstruction.[3]

\section{DIFFERENT TRANSFORM TECHNIQUES}

\subsection{Discrete Fourier Transform:[1]}

Discrete Fourier Transform is a fundamental transform in digital signal processing with applications in frequency analysis, signal analysis etc.

The uth DFT coefficient of length $\mathrm{N}$ sequence $\{\mathrm{f}(\mathrm{x})\}$ is defined as in Equation 3.1: [1]

$$
f(u)=\sum_{x=0}^{N-1} f(x) e^{-\frac{j 2 \Pi u x}{N}}
$$

Equation 3.1

Where $\mathrm{u}=0,1, \ldots \ldots \ldots \ldots \mathrm{N}-1$

Whereas its inverse transform (IDFT) as in Equation 3.2:

$f(x)=\frac{1}{N} \sum_{u=0}^{N-1} F(u) e^{\frac{j 2 \Pi u x}{N}}$

Where $\mathrm{x}=0,1, \ldots \ldots \ldots \ldots \mathrm{N}-1$

The number of complex multiplications and additions to compute DFT is N2.

\subsection{Discrete Cosine Transform:[1]}

The Discrete Cosine Transform is closely related to DFT but only uses only real numbers, it express a function or a signal in terms of a sum of sinusoid with different frequencies and amplitudes. DCT is often used in signal and image processing, especially for lossy data compression, because it has a strong "energy compaction" property .Signal can be reconstructed fairly accurately from only few DCT coefficients.

A discrete Cosine Transform of $\mathrm{N}$ sample is defined as in equation 3.3: [1]

$F(u)=\sqrt{\frac{2}{N}} C(u) \sum_{u=0}^{N-1} f(x) \cos \left(\frac{\Pi(2 x+1) u}{2 N}\right)$
Equation 3.3

Where $\mathrm{u}=0,1, \ldots \ldots \ldots \ldots \mathrm{N}-1$

The inverse DCT is shown in equation 3.4: [1]

$f(x)=\sqrt{\frac{2}{N}} \sum_{u=0}^{N-1} C(u) F(u) \cos \left(\frac{\Pi(2 x+1) u}{2 N}\right)$

Equation 3.4

Where $\mathrm{x}=0,1, \ldots \ldots \ldots \ldots \mathrm{N}-1$

\subsection{Discrete Wavelet Transform:[1]}

Wavelets allow both time and frequency analysis of signals simultaneously because of the fact that energy of wavelet is concentrated in time and still possesses the wave like characteristics. Therefore it is suitable for the analysis of timevarying non-stationary signals such as ECG.

Wavelets are functions generated by one single basis function, called mother wavelet $\psi(t)$ by dilation ' $a$ ' and translation ' $b$ ' represented as in equation 3.5:[1]

$$
\psi_{a, b}(t)=\frac{1}{\sqrt{|a|}} \psi\left(\frac{t-b}{a}\right)
$$

Where $\mathrm{a}=$ scaling parameter and $\mathrm{b}=$ shifting parameter

\section{DIFFERENT COMPRESSION PARAMETERS}

\subsection{Compression Ratio (CR):}

The compression ratio (CR) is defined as the ratio of the number of bits representing the original signal to the number of bits required to store the compressed signal. All data compression algorithm, used to minimize data storage by eliminating the redundancy wherever possible to increase the compression ratio. Compressed data must also represent the data with better fidelity while achieving high compression ratio [2].

Compression Ratio (CR) $=\frac{\text { Bit rate of Original ECG Signal }}{\text { Bit rate of Compressed ECG Signal }}$

A high compression ratio is typically desired. [2]

\subsection{Percentage Root Mean Difference (PRD):}

Percentage root mean difference (PRD) measures distortion between the original signal and the reconstructed signal. 
PRD can be defined as:

$\mathrm{PRD}=\sqrt{\frac{\sum_{n=1}^{N}[x 1(n)-x 2(n)]^{2}}{\sum_{n=1}^{N} x 1(n)^{2}}} * 100$

Where $\mathrm{x} 1(\mathrm{n})=$ original signal of length $\mathrm{N}$ and $\mathrm{x} 2(\mathrm{n})=$ reconstructed signal of length $\mathrm{N}$

The PRD which is an error measurement indicates reconstruction fidelity by point wise comparison with the original data. [2]

\section{COMPRESSION ALGORITHM:[2]}

Step 1: Loading of ECG signal from MIT-BIH arrhythmia data base (record 100)

Step 2: Transform the original ECG signal using DWT

Step 3: To achieve an adaptive threshold compute the maximum value of the transformed coefficients.

Step 4: Apply the threshold of a fix percentage based on absolute maximum values of the transform coefficients.

Step 5: Apply inverse transform to get the reconstruct signal.

Step 6: Calculation of Percent Root Mean Square Difference (PRD).

Step 7: Calculation of Compression Ratio (CR).

\section{DATA COLLECTION}

Loading the ECG signal. Database collection is the one of the most important part of signal processing. MIT-BIH arrhythmia Database directory is selected from physionet. The ECG signals are sampled at $360 \mathrm{~Hz}$ (i.e. 360 samples/second) and each sample is represented by 11 bits/sample. In this work, record 100 of duration 1 minute is taken i.e. 1 second gives 360 samples, 10 seconds gives 3600 samples and 60 seconds (1 minute) gives 21600 samples. Compression algorithm is tested for 1024, 2048, 4096, 8192, 16384 samples of record 100 from MIT-BIH arrhythmia Database.

\section{RESULTS}

Figure 1 (a) shows the original ECG signal and (b) shows the reconstructed signal for 1024 samples of record 100 from MIT-BIH arrhythmia database with Threshold $\lambda=280.7$, Compression Ratio $=34$, Percentage Root Mean Deviation $=$ $2.37 \%$

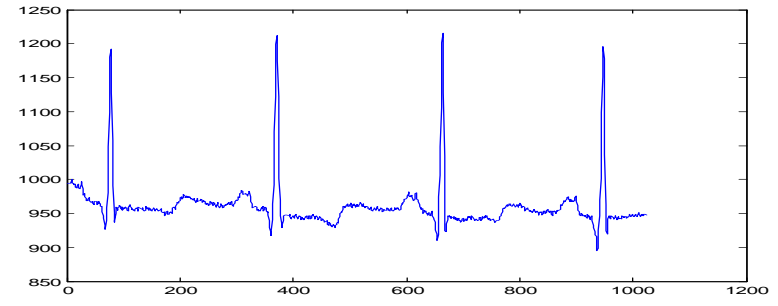

Figure 1(a) Original ECG Signal

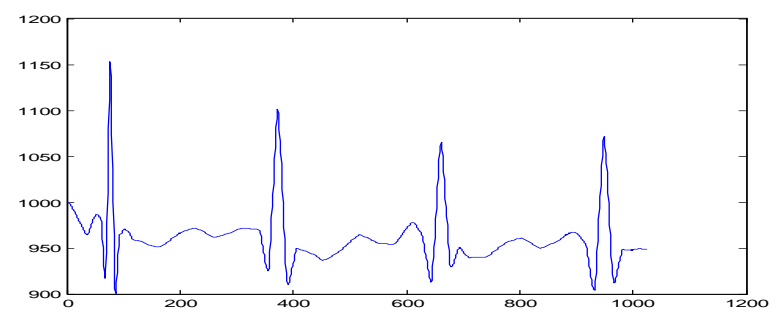

Figure 1(b) Reconstructed ECG Signal

In a similar way, different samples of ECG signal are taken from MIT- BIH arrhythmia data base (record 100) and for a particular threshold value $(\lambda)$, the Compression Ratio (CR) and the error parameter i.e. Percentage Root Mean Deviation (PRD) is calculated.

Table 1 shows the different ECG samples of record 100 and the calculated values of compression ratio and percentage root mean deviation.

Table 1 Different ECG samples and the calculated parameters

\begin{tabular}{|c|c|c|c|}
\hline \multirow{2}{*}{$\begin{array}{c}\text { Number } \\
\text { of } \\
\text { samples }\end{array}$} & Threshold & $\begin{array}{c}\text { Discrete Wavelet Transform } \\
\text { ( Biorthogonal 4.4 wavelet - level 5) }\end{array}$ \\
\cline { 2 - 4 } & Ratio (CR) & $\begin{array}{c}\text { Percentage } \\
\text { Root Mean } \\
\text { Deviation } \\
\text { (PRD in \%) }\end{array}$ \\
\hline 1024 & 280.7 & 34 & 2.37 \\
\hline 2048 & 251.3 & 28.05 & 2.02 \\
\hline 4096 & 252 & 30.1 & 2.068 \\
\hline 8192 & 239.8 & 31.03 & 2.047 \\
\hline 16384 & 267 & 31.5 & 2.25 \\
\hline
\end{tabular}

Figure 2 (a) shows the original ECG signal and (b) shows the reconstructed signal for 16384 samples of record 100 from MIT-BIH arrhythmia database with Threshold $\lambda=267$, Compression Ratio $=31.5$, Percentage Root Mean Deviation $=$ $2.25 \%$ 


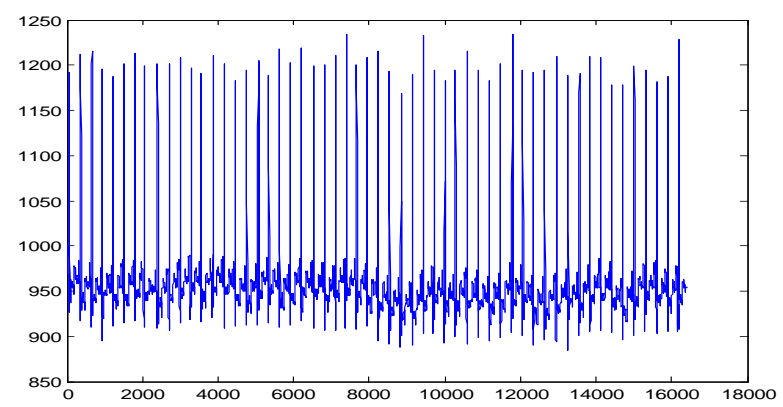

Figure 2(a) Original ECG Signal

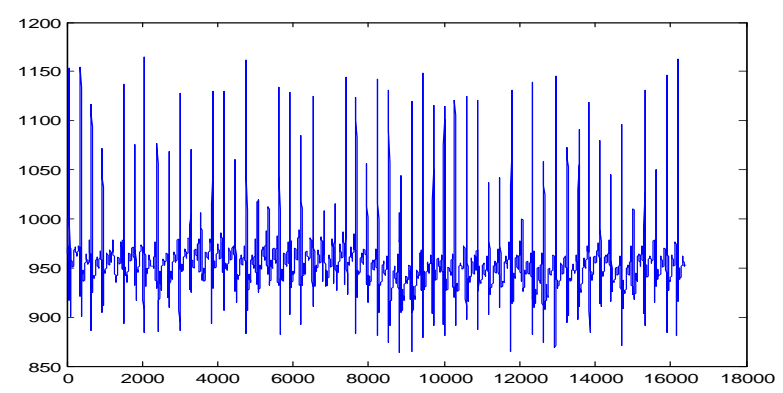

Figure 2(b) Reconstructed ECG Signal

\section{CONCLUSIONS}

From the results it can be concluded that as threshold value $(\lambda)$ increases, the Compression Ratio (CR) increases and the error criterion Percentage Root Mean Deviation (PRD) value also increases which yields high data reduction and poor signal fidelity. Low threshold value gives low data reduction and high signal fidelity. So threshold value should be selected such that the quality of the ECG signal is not distorted on reconstruction and a good amount of data reduction is also achieved. The same data compression algorithm is to be implemented in FPGA using Verilog HDL.

\section{REFERENCES:}

[1] Mrs.S.O.Rajankar and Dr. S.N. Talbar, "An Optimized Transform for ECG Signal Compression", ACEEE Int. J. on Signal \& Image Processing, Vol. 01, No. 03, Dec 2010

[2] Ruqaiya Khanam and Syed Naseem Ahmad," ECG Signal Compression for Diverse Transforms", ISSN 2224-5758 (Paper) ISSN 2224-896X (Online, Vol 2, No.5, 2012

[3] Prof. Mohammed Abo-Zahhad," ECG Signal Compression Using Discrete Wavelet Transform", Vice-Dean for Graduate Studies, Faculty of Engineering, University of Assiut, Egypt [4] Hari Mohan Rai and Anurag Trivedi, "De-noising of ECG Waveforms based on Multi-resolution Wavelet Transform", International Journal of Computer Applications (0975 - 8887)

Volume 45- No.18 May 2012
[5] Chengjun Zhang and Chunyan Wang, "A Pipeline VLSI Architecture for High-Speed Computation of the 1-D Discrete Wavelet Transform", IEEE TRANSACTIONS ON CIRCUITS AND SYSTEMS-I: REGULAR PAPERS, VOL. 57, NO. 10, OCTOBER 2010

[6] M. JANARDAN and Dr. K ASHOK BABU, "AN EFFICIENT ARCHITECTURE FOR 3-D LIFTING-BASED DISCRETE WAVELET TRANSFORM", M Janardan et al, Int. J. Comp. Tech. Appl., Vol 2 (5), 1439-1458

[7] Mohammed Abo-Zahhad, SabahM. Ahmed, and Ahmed Zakaria, "An Efficient Technique for Compressing ECG Signals Using QRS Detection, Estimation, and 2D DWT Coefficients Thresholding", Hindawi Publishing Corporation Modelling and Simulation in Engineering,Volume 2012, Article ID 742786, 10 pages doi:10.1155/2012/742786

[8] Lecture Notes: Ali Shoeb and Gari Clifford, "Chapter 16 Wavelets; Multiscale Activity in Physiological Signals", HST582J/6.555J/16.456J Biomedical Signal and Image Processing Spring 2005

[9] Apoorv Gautam1 and Maninder Kaur, "ECG Analysis using Continuous Wavelet Transform (CWT)", IOSR Journal of Engineering Apr. 2012, Vol. 2(4) pp: 632-635

[10] MIT- BIH arrhythmia data base www.physionet.org

[11] www.mathworks.com

[12] www.intechopen.com

\section{BIOGRAPHIES:}

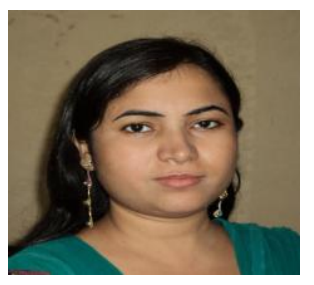

Karishma Qureshi received the B. E. degree in Electronics and Communication in 2003. She is working as an Engineer-SD at Institute for Plasma Research since 2005. She is pursuing her Masters in Engineering from L. D. College of Engineering in Applied Instrumentation Department, Ahemdabad

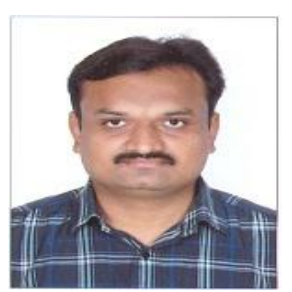

Vinod Patel received M. Tech degree from Indian Institute of Technology, Roorkee in the year 2007. Currently he is working as an Associate Professor and Head in Applied Instrumentation Department at L. D. College of Engineering, Ahemdabad 